\title{
Models of Impulsivity with a Focus on Waiting Impulsivity: Translational Potential for Neuropsychiatric Disorders
}

\author{
Valerie Voon \\ Published online: 3 October 2014 \\ (C) The Author(s) 2014. This article is published with open access at Springerlink.com
}

\begin{abstract}
Waiting impulsivity, also known as premature or anticipatory responding, is well established in preclinical studies through the 5-Choice Serial Reaction Time (5-CSRT) task. Waiting impulsivity is important in disorders of addiction. Preclinical studies suggest a role both as a predictor, and as a consequence, in disorders of addiction. Here we discuss the relationship between the preclinical 5-CSRT and translational fidelity in newly developed translational tasks. Preclinical and clinical literature relevant to premature responding and disorders of addiction are reviewed. Understanding which processes are critical to premature responding is important in understanding the nature of premature responding. Premature responding may also have overlaps with motivational processes, proactive response inhibition, tonic inhibitory processes, and delay discounting.
\end{abstract}

Keywords Waiting impulsivity · Premature responding · Impulsivity $\cdot$ Addiction $\cdot 5$-CSRT

\section{Introduction}

Waiting impulsivity, also known as premature or anticipatory responding or impulsive action, is well characterized in preclinical rodent studies and more recently translated in human studies. Waiting impulsivity has potentially important clinical implications in disorders of addiction, as preclinical evidence suggests it can play a role both as a predictor, and as a consequence of disorders of addiction. Impulsivity is generally defined as the

V. Voon $(\bowtie)$

Department of Psychiatry, University of Cambridge, Cambridge CB2 0QQ, UK

e-mail: voonval@gmail.com

V. Voon

Behavioural and Clinical Neuroscience Institute, University of Cambridge, Cambridge, UK

V. Voon

Cambridgeshire and Peterborough NHS Foundation Trust,

Cambridge, UK predisposition to act with a low or inadequate degree of deliberation, forethought, or control [1]. Waiting impulsivity is a subtype of impulsivity defined operationally as the tendency to respond before target onset, and can be assessed in rodents using the 5-Choice Serial Reaction Time Task (5-CSRTT) [2••]. The broader concept of impulsivity is a heterogeneous construct, which can be subdivided into: waiting impulsivity; motor response inhibition (the ability to cancel or restrain a pre-potent motor response); and decisional forms, including delay discounting (the tendency to select an immediate smaller reward over a larger delayed reward); or reflection impulsivity (the tendency towards rapid decisions, without adequate accumulation or consideration of the evidence). These subtypes have overlapping yet dissociable neural substrates [3]. Here we focus on evidence for preclinical and clinical evidence of waiting impulsivity, and its relationship to disorders of addiction. We first discuss the rodent 5-CSRTT and the role of state and trait effects in disorders of addiction. A brief summary of neural and neurochemical correlates from the preclinical literature is reviewed. This review focuses predominantly on newly developed translational tasks for humans including the 4-Choice Serial Reaction Time Task (4-CSRTT), which was developed to maintain translational fidelity with the 5-CSRTT. We then discuss cognitive processes that may contribute to further understanding the nature of human premature responding.

\section{Preclinical}

\section{5-Choice Serial Reaction Time Task}

Here we describe the rodent 5-CSRTT for the purposes of comparison with the human task. In the preclinical 5CSRTT, rodents are placed in a box with five apertures and wait for a brief 0.5 second light cue or target predicting reward to appear behind one of the apertures before performing a nose-poke at the aperture. Accurate responding is rewarded with a food pellet collected at the food dispenser. Premature responding is measured as an early nose-poke before cue or target 
presentation and is punished by a five second time-out (house light extinguished and no delivery of food reward) (Robbins 2001). A failure to respond during the limited hold (a specified time interval after target onset and time for subject to respond), is recorded as an omission and punished by a five second time-out. Responses at a non-illuminated aperture are recorded as incorrect responses and are also punished by a five second time out. Repeated responding at the aperture is recorded as perseverative responding. The accuracy rate of target detection is calculated as the percentage of correct responses to the total number of correct and incorrect responses. Rodents are typically trained extensively on a fixed inter-trial interval (e.g., five second duration between food collection and light or target onset), with testing occurring on a prolonged inter-trial interval (e.g., seven seconds).

\section{State and Trait Effects}

Premature responding has both trait (an endophenotypic predictor or risk factor for the development of a disorder), and state (a secondary consequence of exposure), effects dependent on the substance. Both stimulants and nicotine have preclinical evidence for both state and trait effects. High levels of premorbid premature responding in rodents predicts greater addiction-like behavior to cocaine defined operationally as: increased motivation to take cocaine; inability to inhibit drug seeking; and continued drug use despite aversive consequences [4•]. Greater premature responding for up to two weeks of abstinence, following chronic methamphetamine exposure, suggests premature responding can also be secondary to methamphetamine exposure [5]. In rodents, high premorbid premature responding predicts greater motivation to initiate and maintain nicotine use [6•], and nicotine increases premature responding [7].

Evidence for a role for premorbid impulsivity in predicting the development of alcohol use disorders is less clear. Premature responding is associated with alcohol-preferring mice strains as compared to non-alcohol preferring strains [8•], and is also associated with greater withdrawal severity from chronic alcohol amongst different mice strains [9]. Acute alcohol exposure [10], and early (but not late), abstinence following chronic alcohol exposure, are also associated with increased premature responding in rodents [11], suggesting that premature responding can be secondary to both acute and chronic alcohol exposure. High premorbid premature responding is also associated with greater escalation of sucrose-seeking behavior and reinstatement following extinction in rodents [12•]. The association between premature responding and strain differences is less clear, as premature responding in 15 different strains of mice is not associated with sucrose acquisition or preference [9].

Neural Networks and Neurochemistry

In rodents, premature responding implicates a network including the nucleus accumbens, infralimbic cortex, and subthalamic nucleus. Dopaminergic, noradrenergic, serotonergic, and GABA-ergic mechanisms have also been implicated in premature responding. The evidence for a role for these neural regions and neurochemistry in premature responding is briefly reviewed.

Acute amphetamine increases premature responding in rodents, an effect attenuated by 6-hydroxydopamine lesions of the nucleus accumbens, and by D1/2 receptor antagonists [13]. High impulsive rodents have lower ventral striatal D2/3 receptor availability [14], and have lower left grey matter density in the nucleus accumbens core [15]. Atomoxetine, a selective norepinephrine-reuptake inhibitor, also dosedependently decreases premature responding [16]. An opponency effect has been observed in the nucleus accumbens core and shell: microinfusion of methylphenidate, a mixed dopamine/norepinephrine reuptake inhibitor, in the core (but not the shell), is associated with increased premature responding [17]. In contrast, microinfusions of atomoxetine into the shell (but not the core), decreases premature responding. In rodents, infusion of quinpirole (a D2/3 agonist), into the nucleus accumbens core increases premature responding in high impulsive rodents, but within the shell increases locomotor activity [18]. High impulsive rodents also had decreased nucleus accumbens core glutamate decarboxylase (GAD 65/67), an enzyme implicated in the conversion of glutamate to GABA [15]. Furthermore, experimentally decreasing mRNA gene expression of GAD 65/67 increased impulsivity in low impulsive rodents.

Lesions of the infralimbic cortex (equivalent to the human subgenual anterior cingulate- SgACC), also increase premature responding [19]; although neither methylphenidate, nor atomoxetine, in the infralimbic cortex influence premature responding [17]. Central serotonin depletion and prefrontal 5-HT2C receptor antagonism is also associated with greater premature responding in rodents, whereas prefrontal 5-HT2A receptor antagonism is associated with a decrease in premature responding [20]. Similarly, 5HT2A receptor antagonists have been shown to decrease cocaine-induced premature responding in rodents [21], and $5 \mathrm{HT} 2 \mathrm{C}$ receptor agonists have been shown to decrease nicotine-induced premature responding [22]. Lesions of the subthalamic nucleus in rodents are also associated with a robust enhancement in premature responding $[23,24]$.

\section{Clinical}

Premature Responding Tasks

There are several existing tasks assessing anticipatory, early or premature responding that have been tested in humans including the 4-CSRTT, Continuous Performance Task, Traffic Light task, Simon task and Sx-5CSRTT. 


\section{4-Choice Serial Reaction Time Task (4-CSRT)}

The 4-CSRT task was developed based on the rodent 5-CSRT. Subjects are seated in front of a touch screen [25••]. When four boxes appear on the screen, the subject presses and holds down the space bar on the keyboard with their dominant index finger indicating the 'cue onset' time. After a specified period (cue-target interval), a green circle target appears briefly and randomly in one of the four boxes. Subjects release the space bar and touch the box in which the target appeared. Baseline blocks without monetary feedback are used to individualize monetary feedback amounts for subsequent blocks, based on the individual's mean fastest reaction time and standard deviation. The four Test blocks with monetary feedback were optimized, to increase premature responding, and varied by target duration and variability of the cue-target interval and the presence of distractors. Accurate and timely responses were followed by different reward magnitude outcomes, depending on the speed of responding. The primary outcome measure is the premature release of the space bar prior to target onset. Following a premature release, subjects were required to complete the trial by touching the screen, which was followed by a feedback slide indicating to 'Keep going,' without reward (equivalent to a time out penalty). Incorrect responses are errors of commission (wrong box touched after target onset). Other outcome measures included accuracy (correct responses/ correct + incorrect responses); late responses (late responses/correct fast responses + late responses, corresponding to errors of omission in the rodent paradigm); and total amount won.

Using the 4-CSRTT, our research group has shown that relative to healthy volunteers, premature responding is elevated in subjects abstinent from alcohol and methamphetamine use disorders, recreational cannabis users, and in current (but not ex- or non-), smokers [25••]. The observation in smokers suggests either a role for premature responding as a consequence of nicotine or that those with lower premature responding may be more effective at abstinence. There were no differences in premature responding observed in obese subjects, with or without, binge eating disorder. However, this may be related to testing in the context of satiety rather than food deprivation; use of a conditioned reinforcer (monetary outcome), rather than a primary reinforcer (food); or testing subjects with lower levels of obesity, rather than morbid obesity. Using this same task, we have also shown that premature responding in humans increases with tryptophan depletion [26 ], or with a decrease in central serotonin levels.

We have further shown that premature responding in humans correlates negatively with increasing age, consistent with the trajectory of impulsivity with age [27]. Although we did not show a correlation between premature responding and impulsivity questionnaires in one study [25••], the tryptophan depletion study did show a correlation with impulsivity questionnaires
[26•]. Premature responding did not correlate with IQ, depression scores, accuracy, reaction time, late responses, amount of money won, or motivation for monetary feedback $[25 \bullet \bullet]$.

\section{Continuous Performance Task}

The original rodent 5-CSRTT was developed based on a human serial reaction time task or continuous performance task (CPT). A more demanding version of the CPT, the Immediate and Delayed Memory Task (IMT/DMT), has been used to measure impulsive action in a cross-species translational study $[28,29]$. Rodents were tested on the 5-CSRTT with both amphetamine and atomoxetine manipulation, and humans were tested on the IMT/DMT. The IMT/DMT consists of randomly generated five-digit numbers. In the IMT, subjects respond to the target stimuli when two identical randomly generated five-digit numbers are presented in sequence. In the DMT, a distracter stimulus (e.g., 12345), appears three times between the target stimuli and should be ignored. Impulsive action errors were measured as the ratio of commission errors (responses to non-identical numbers), to correct detections; or the equivalent action restraint errors in go/no go tasks. Although this task focuses on commission errors, as discussed below in the section on processes related to waiting impulsivity, the role of response inhibition prior to motor initiation may be of relevance in premature responding.

\section{Traffic Light Task}

In the Traffic Light task, subjects respond to optimize timesensitive risky rewarding outcomes [30,31•]. Subjects view a red light that turns amber and then green, and must respond rapidly to the green Go signal to obtain a reward. The reward value is greatest at the minimum possible latency following the Go signal, and declines steeply with increasing reaction time after Go signal onset. The amber duration is variable so the time of onset of the Go signal cannot be predicted. To optimize the reward, rather than waiting for the Go signal and responding reactively, subjects should respond during the anticipatory amber period prior to Go signal onset. Such a response has an element of a risk estimation and choice, since the onset of the Go signal cannot be accurately predicted: a successful motor response immediately after the Go signal is optimally rewarded, but responding prior to the Go signal is penalized with a small fixed penalty. The outcome measure is the frequency of penalized trials and the overall reward obtained. Anticipatory responding, measured using this task, correlated with the lack of premeditation on the UPPS Impulsive Behaviour Scale, and was negatively correlated with age in healthy volunteers [30]. The anticipatory response in the Traffic Light task thus occurs in the context of a risky choice, response selection, sensitivity to reward and loss, and capacity for time estimation. 


\section{Simon Task}

Anticipatory responding has also been reported using the Simon task, in which an irrelevant stimulus evokes an early strong response impulse that interferes with goal-directed action; with subsequent response inhibition affecting later impulsive actions $[32 \bullet \bullet, 33,34]$. In the Simon task, subjects view a central fixation point followed by a blue or green circle appearing to the left or right of the fixation point. Subjects respond with either the left or right finger, dependent on an indicated colour response mapping. In corresponding trials the spatial location of the circle matches with the colour response mapping (e.g., blue circle mapped to right finger appears on right). In noncorresponding trials, the spatial location of the circle was opposite that of the colour response mapping (e.g., blue circle mapped to right finger appears on left). The analysis is based on an activation-suppression model in which accuracy is parcellated into reaction time bins; thus differentiating early stimulus-driven, prepotent, impulsive, but erroneous actions; and subsequent later selective suppression of these impulsive actions, to facilitate selection of the correct action. In this task, the early erroneous motor response occurs after the stimulus onset and does not specifically address anticipatory or waiting impulsivity. The impulsive response is driven by the irrelevant stimulus capturing a prepotent response, which can either facilitate or interfere with the actual goal-oriented response. The task thus assesses prepotent stimulus-induced impulsive responding in the context of conflict, and dissociates this measure from the influence of response suppression and response selection.

This early impulsive response in healthy volunteers is associated with activity in the pre-supplementary motor area (pre-SMA), and the late response with right inferior frontal cortex activity [34]. Impairment of the pre-SMA using transcranial magnetic stimulation in the Simon task decreased impulsive responses, particularly when subjects were being explicitly rewarded for fast accurate responses suggesting an influence of motivation or reward anticipation [35]. The authors suggest a parallel influence of prefrontal regions, since pre-SMA impairment was associated with greater connectivity of the inferior frontal gyrus and subthalamic nucleus, along with decreased impulsivity. Subthalamic deep brain stimulation in Parkinson's disease patients has been shown to enhance early stimulus-driven impulsive responding in the Simon task, but to improve selective inhibition of late responses [32••]. Parkinson's patients with medication-induced impulse control behaviors also had less early impulsive responding, relative to those without impulse control behaviors [36], suggesting this form of impulsivity is not relevant to the disorder.

\section{Sussex-5-Choice Serial Reaction Time Task}

In the Sussex-5-Choice Serial Reaction Time task (Sx5CRST), subjects respond to a highlighted stimulus from one of five moving stimuli using their dominant hand [8•]. Premature responding is measured as a response prior to onset of the highlighted stimulus, and penalized by a five second time out. Both fixed and variable interval conditions are tested along with both conditions under a dual-task condition, with responding with a key press to a tone with the non-dominant hand. Subjects are not explicitly rewarded for correct responding. Thus, the task may focus more on motoric impulsivity elements, rather than on impulsivity in the context of reward anticipation.

Using this task, male binge drinking subjects had higher premature responding, compared to healthy males in the fixed interval condition [8•]. In the distraction condition, binge drinkers of both genders were associated with greater premature responding, along with a greater number of errors relative to healthy volunteers, suggesting a role for attentional load or task difficulty in mediating these findings.

\section{Summary}

Several tasks have been developed to assess waiting impulsivity in human subjects, some of which may be influenced by other features including response inhibition, anticipatory responding in the context of risk, stimulus-induced prepotent responses, and the role of reward anticipation.

Processes that Might Influence or are Related to Waiting Impulsivity

Waiting impulsivity as measured using the rodent 5CSRT assesses premature responding in the context of anticipation of cues predicting reward. There are several cognitive processes that may play a role in premature responding which include attention, motivation, delay discounting, response inhibition, sensitivity to reward, and to negative outcomes.

\section{Attention}

The accuracy measure in the 4-CSRT accounts in part for the non-specific influences such as attentional capacity, motivation, or motor behavior, since both correct and incorrect responses require the same motor effort $[2 \bullet \bullet]$. We did not show any correlation between accuracy measures and premature responding, suggesting non-specific attention can be dissociated from premature responding [25••]. Under tryptophan depletion, we show enhanced premature responding in humans as tested using the 4-CSRT, along with enhanced accuracy, suggesting that attentional deficits are unlikely to account for the increase in premature responding [26•]. 


\section{Sensitivity to Negative Outcomes}

Both the 4-CSRT and Sx-5CSRT have time out penalties following a premature response. During task development of the 4-CSRT, the introduction of an explicit monetary loss feedback to the premature response resulted in a marked decrement in premature responding in healthy volunteers. Sensitivity to negative feedback may be relevant in disorders of addiction, as rodent models of binge drinking and human binge drinking subjects have shown decreased sensitivity to aversive reinforcement [37]. Our group has also shown that young-adult BD subjects are more risk-seeking when anticipating loss outcomes, similarly suggesting impaired sensitivity to aversive outcomes [38].

\section{Motivational Processes}

One possible relevant mechanism is that of a generalized enhancement in incentive motivational responding. The 4CSRT has, embedded within the design, a means of assessing reaction time; either as an index of motivational responding, or reward sensitivity. Subjects first learn to respond as quickly as possible to the target in Baseline 1, used to individualize reward feedback. Following Baseline 1, Test Block 1 has a similar design as Baseline 1, although responding to the target is now followed by monetary feedback. A second baseline block, Baseline 2 (equivalent to Baseline 1), follows Test Block 1. Thus, Baseline 2 follows instrumental responding to a target with monetary feedback, consistent with testing in extinction without feedback. The Motivation Index, or difference between the baseline blocks, might thus allow the assessment of a change in reaction time, as an index of motivation or reward sensitivity; testing either differences in responding to over-learned instrumental goal-directed behaviors tested in extinction, or if the target becomes conditioned to the reward, then testing responding to the conditioned stimulus in extinction.

Using the 4-CSRT we show that Motivation Index, which was unrelated to premature responding, is decreased in abstinent alcohol-dependent subjects, and is negatively correlated with both the severity of alcohol dependence and binge eating $[25 \bullet \bullet$.

\section{Delay Discounting}

In the rodent literature, premature responding correlates with delay discounting [39], suggesting overlaps in the impairment of 'waiting' for reward. However, in the human 4-CSRT, delay discounting as tested using the hypothetical Monetary Choice Questionnaire was not correlated with premature responding [25••]. There are several reasons we might observe such a dissociation.
The neural substrates of premature responding and delay discounting overlap particularly in the ventral striatum, but are not identical. Lesions of the rodent nucleus accumbens core, amygdala, and hippocampus increase delay discounting, suggesting that these structures are critical to the evaluative process [40-42]. In human fMRI studies, the ventral striatum, dorsolateral prefrontal cortex, insula, amygdala, posterior cingulate, and parietal cortex have been implicated in delay discounting for secondary [43-46], and primary rewards [47]. The orbitofrontal cortex is also implicated in timediscounting of rewards [48], with lesions of the rodent medial and lateral orbitofrontal cortex associated with a decrease and increase in delay discounting respectively [49]; and orbitofrontal cortex lesions in humans is associated with greater delay discounting [50].

Delay discounting in rodents is typically tested by determining the indifference point between an immediate choice of a small reward and a delayed choice of a larger reward, by manipulating the duration of the delay or the reward magnitude. Rodent studies use reward feedback in real-time over short delays (e.g., in seconds), whereas the Monetary Choice Questionnaire in humans uses hypothetical monetary feedback over long delays (e.g., in days equivalent to weeks and months). Discounting tasks in real time with feedback such as the Experiential Discounting Task have been developed for human studies, and might show a different relationship with premature responding [51]. The short duration for the delayed reward has also been shown in human studies to differ from longer delays by being better modeled by an exponential, rather than a hypothetical, discount function [52]. What aspect of delay discounting might be related to premature responding is also relevant, as delay discounting itself can be parcellated into several cognitive mechanisms, including (but not limited to), the incentive salience of the immediate choice, the discounting effects of the delayed reward, delay aversion, the uncertainty of the delayed choice, magnitude effects, time estimation or decreasing subjective valuation of increasing magnitude.

\section{Response Inhibition}

Response inhibition may be implicated in premature responding, particularly in the context of tonic inhibitory mechanisms and proactive stopping or stopping prior to initiation of a motor action. The stop signal task assesses action cancellation of a prepotent motor action that has already started, whereas the Go/NoGo task assesses action restraint of a prepotent motor action that is not yet initiated, the latter of which may be more relevant. In the rodent 5CSRTT, differences between premature responding and false errors (analogous to Go/NoGo commission errors), have been highlighted [53]. In rodent and human studies, there is no relationship 
between premature responding and the stop signal task [25••], but the relationship with the more relevant role of action restraint in humans has not yet been tested. The neural substrates of the stop signal and Go/NoGo tasks overlap and implicate the pre-SMA, right inferior frontal gyrus, caudate, and subthalamic nucleus (STN) [54-56]. These neural regions overlap with premature responding particularly in the STN.

Proactive stopping [57], or preparing to suppress a response tendency rather than reactive stopping after signal onset, may be relevant to premature responding. The premature responding task does not include external stop signals. When stop signals are expected and introduced in a task, behavioral responses change with proactive response strategies implemented, thus increasing response thresholds, slowing the go reaction time, and increasing the likelihood of stopping $[57,58]$. Proactive stopping with internal, rather than external, signals may also be relevant in premature responding. The STN is hypothesized to be engaged in influencing response suppression by inhibition of thalamocortical pathways via both reactive and proactive models. In reactive stopping, the STN is proposed to relay a reactive global No-Go signal to inhibit execution during high conflict tasks, thus allowing greater time to integrate information [59, 60]. Alternatively, in the proactive inhibition model of the STN, inhibitory processes are suggested to generalize in which the default state is a tonic inhibitory process involved in the suppression of automatic or prepotent responses. This proactive stopping and tonic inhibitory process may be relevant to premature responding in the context of prepotent reward anticipation.

\section{Conclusion}

Waiting impulsivity is an important process in disorders of addiction. Preclinical studies suggest a role both as a predictor and as a consequence in disorders of addiction. We suggest that one of the human analogues, the 4-CSRT, has strong translational fidelity with the rodent version of the 5-CSRT task. Which processes are the elements crucial to premature responding is important in understanding the nature of premature responding. These include impulsivity, in the context of the anticipation of a cue predicting reward, the role of nonspecific anticipatory motor responding, responding in the context of an irrelevant stimulus inducing a prepotent response, or in the context of risk. The relationship between premature responding and motivational processes, proactive response inhibition, tonic inhibitory processes, and delay discounting remain to be further studied. An in depth understanding of the nature of premature responding in humans allows us to develop more specific targeted behavioral measures and interventions.
Acknowledgments Dr. Voon is a Wellcome Trust Fellow and supported by the Wellcome Trust (093705/Z/10/Z).

\section{Compliance with Ethics Guidelines}

Conflict of Interest Valerie Voon declares no conflict of interest.

Human and Animal Rights and Informed Consent This article does not contain any studies with human or animal subjects performed by any of the authors.

Open Access This article is distributed under the terms of the Creative Commons Attribution License which permits any use, distribution, and reproduction in any medium, provided the original author(s) and the source are credited.

\section{References}

Papers of particular interest, published recently, have been highlighted as:

- Of importance

•. Of major importance

1. Moeller FG, Barratt ES, Dougherty DM, Schmitz JM, Swann AC. Psychiatric aspects of impulsivity. Am J Psychiatry. 2001;158:1783-93.

2.・ Robbins TW. The 5-choice serial reaction time task: behavioural pharmacology and functional neurochemistry. Psychopharmacology (Berl). 2002;163:362-80. Classic review of the development of the rodent 5-Choice Serial Reaction Time task.

3. Dalley JW, Everitt BJ, Robbins TW. Impulsivity, compulsivity, and top-down cognitive control. Neuron. 2011;69:680-94.

4. Belin D, Mar AC, Dalley JW, Robbins TW, Everitt BJ. High impulsivity predicts the switch to compulsive cocaine-taking. Science. 2008;320:1352-5. Premorbid high waiting impulsivity predicts compulsive cocaine seeking behaviours.

5. Dalley JW, Laane K, Theobald DE, Pena Y, Bruce CC, Huszar AC, et al. Enduring deficits in sustained visual attention during withdrawal of intravenous methylenedioxymethamphetamine selfadministration in rats: Results from a comparative study with damphetamine and methamphetamine. Neuropsychopharmacology. 2007;32:1195-206.

6. Diergaarde L, Pattij T, Poortvliet I, Hogenboom F, de Vries W, Schoffelmeer AN, et al. Impulsive choice and impulsive action predict vulnerability to distinct stages of nicotine seeking in rats. Biol Psychiatry. 2008;63:301-8. Premorbid high waiting impulsivity predicts nicotine seeking.

7. Blondel A, Sanger DJ, Moser PC. Characterisation of the effects of nicotine in the five-choice serial reaction time task in rats: Antagonist studies. Psychopharmacology (Berl). 2000;149:293-305.

8. Sanchez-Roige S, Baro V, Trick L, Pena-Oliver Y, Stephens DN, Duka T. Exaggerated waiting impulsivity associated with human binge drinking, and high alcohol consumption in mice. Neuropsychopharmacology. 2014. Sussex 5-CSRT: high waiting impulsivity in human binge drinking and alcohol prefering mouse strains.

9. Gubner NR, Wilhelm CJ, Phillips TJ, Mitchell SH. Strain differences in behavioral inhibition in a go/no-go task demonstrated using 15 inbred mouse strains. Alcohol Clin Exp Res. 2010;34: $1353-62$.

10. Oliver YP, Ripley TL, Stephens DN. Ethanol effects on impulsivity in two mouse strains: similarities to diazepam and ketamine. Psychopharmacology (Berl). 2009;204:679-92. 
11. Walker SE, Pena-Oliver Y, Stephens DN. Learning not to be impulsive: disruption by experience of alcohol withdrawal. Psychopharmacology (Berl). 2011;217:433-42.

12. Diergaarde L, Pattij T, Nawijn L, Schoffelmeer AN, De Vries TJ. Trait impulsivity predicts escalation of sucrose seeking and hypersensitivity to sucrose-associated stimuli. Behav Neurosci. 2009;123:794-803. Premorbid waiting impulsivity predicts sucrose seeking.

13. Cole BJ, Robbins TW. Effects of 6-hydroxydopamine lesions of the nucleus accumbens septi on performance of a 5choice serial reaction time task in rats: implications for theories of selective attention and arousal. Behav Brain Res. 1989;33:165-79.

14. Dalley JW, Fryer TD, Brichard L, Robinson ES, Theobald DE, Laane $\mathrm{K}$, et al. Nucleus accumbens $\mathrm{d} 2 / 3$ receptors predict trait impulsivity and cocaine reinforcement. Science. 2007;315:126770.

15. Caprioli D, Sawiak SJ, Merlo E, Theobald DE, Spoelder M, Jupp B, Voon V, Carpenter TA, Everitt BJ, Robbins TW, Dalley JW. Gamma aminobutyric acidergic and neuronal structural markers in the nucleus accumbens core underlie trait-like impulsive behavior. Biol Psychiatry. 2013

16. Robinson ES, Eagle DM, Mar AC, Bari A, Banerjee G, Jiang X, et al. Similar effects of the selective noradrenaline reuptake inhibitor atomoxetine on three distinct forms of impulsivity in the rat. Neuropsychopharmacology. 2008;33:1028-37.

17. Economidou D, Theobald DE, Robbins TW, Everitt BJ, Dalley JW. Norepinephrine and dopamine modulate impulsivity on the fivechoice serial reaction time task through opponent actions in the shell and core sub-regions of the nucleus accumbens. Neuropsychopharmacology. 2012;37:2057-66.

18. Moreno M, Economidou D, Mar AC, Lopez-Granero C, Caprioli D, Theobald DE, et al. Divergent effects of $\mathrm{d}(2) /$ (3) receptor activation in the nucleus accumbens core and shell on impulsivity and locomotor activity in high and low impulsive rats. Psychopharmacology (Berl). 2013;228:1930 .

19. Chudasama Y, Passetti F, Rhodes SE, Lopian D, Desai A, Robbins TW. Dissociable aspects of performance on the 5-choice serial reaction time task following lesions of the dorsal anterior cingulate, infralimbic and orbitofrontal cortex in the rat: differential effects on selectivity, impulsivity and compulsivity. Behav Brain Res. 2003;146:105-19.

20. Winstanley CA, Theobald DE, Dalley JW, Glennon JC, Robbins TW. 5-ht2a and 5-ht2c receptor antagonists have opposing effects on a measure of impulsivity: interactions with global 5-ht depletion. Psychopharmacology (Berl). 2004;176:376-85.

21. Anastasio NC, Stoffel EC, Fox RG, Bubar MJ, Rice KC, Moeller FG, et al. Serotonin (5-hydroxytryptamine) 5-ht(2a) receptor: association with inherent and cocaine-evoked behavioral disinhibition in rats. Behav Pharmacol. 2011;22:248-61.

22. Higgins GA, Silenieks LB, Rossmann A, Rizos Z, Noble K, Soko $\mathrm{AD}$, et al. The 5-ht2c receptor agonist lorcaserin reduces nicotine self-administration, discrimination, and reinstatement: relationship to feeding behavior and impulse control. Neuropsychopharmacology. 2012;37:1177-91.

23. Baunez C, Nieoullon A, Amalric M. In a rat model of parkinsonism, lesions of the subthalamic nucleus reverse increases of reaction time but induce a dramatic premature responding deficit. J Neurosci Off J Soc Neurosci. 1995;15:6531-41.

24. Baunez C, Robbins TW. Bilateral lesions of the subthalamic nucleus induce multiple deficits in an attentional task in rats. Eur J Neurosci. 1997;9:2086-99.

25.• Voon V, Irvine MA, Derbyshire K, Worbe Y, Lange I, Abbott S, et al. Measuring "waiting" impulsivity in substance addictions and binge eating disorder in a novel analogue of rodent serial reaction time task. Biol Psychiatry. 2014;75:148-55. Human waiting impulsivity (4-CSRT) across drug and natural rewards.

26. Worbe Y, Savulich G, Voon V, Fernandez-Egea E, Robbins TW. Serotonin depletion induces 'waiting impulsivity' on the human four-choice serial reaction time task: Cross-species translational significance. Neuropsychopharmacology. 2014;39:1519-26. Tryptophan depletion increases waiting impulsivity in humans.

27. Steinberg L, Albert D, Cauffman E, Banich M, Graham S, Woolard $\mathrm{J}$. Age differences in sensation seeking and impulsivity as indexed by behavior and self-report: evidence for a dual systems model. Dev Psychol. 2008;44:1764-78.

28. Broos N, Schmaal L, Wiskerke J, Kostelijk L, Lam T, Stoop N, et al. The relationship between impulsive choice and impulsive action: a cross-species translational study. PLoS One. 2012;7: e36781.

29. Dougherty DM, Marsh DM, Mathias CW. Immediate and delayed memory tasks: a computerized behavioral measure of memory, attention, and impulsivity. Behav Res Methods Instrum Comput $\mathbf{J}$ Psychon Soc Inc. 2002;34:391-8.

30. Burnett Heyes S, Adam RJ, Urner M, van der Leer L, Bahrami B, Bays PM, et al. Impulsivity and rapid decision-making for reward. Front Psychol. 2012;3:153.

31. Adam R, Bays PM, Husain M. Rapid decision-making under risk. Cogn Neurosci. 2012;3:52-61. Traffic light task.

32.• Wylie SA, Ridderinkhof KR, Elias WJ, Frysinger RC, Bashore TR, Downs KE, et al. Subthalamic nucleus stimulation influences expression and suppression of impulsive behaviour in parkinson's disease. Brain. 2010;133:3611-24. Effects of subthalamic stimulation and activation-suppression model.

33. van den Wildenberg WP, Wylie SA, Forstmann BU, Burle B, Hasbroucq T, Ridderinkhof KR. To head or to heed? Beyond the surface of selective action inhibition: a review. Front Hum Neurosci. 2010;4:222.

34. Forstmann BU, van den Wildenberg WP, Ridderinkhof KR. Neural mechanisms, temporal dynamics, and individual differences in interference control. J Cogn Neurosci. 2008;20:1854-65.

35. Herz DM, Christensen MS, Bruggemann N, Hulme OJ, Ridderinkhof KR, Madsen KH, et al. Motivational tuning of fronto-subthalamic connectivity facilitates control of action impulses. J Neurosci. 2014;34:3210-7.

36. Wylie SA, Claassen DO, Huizenga HM, Schewel KD, Ridderinkhof KR, Bashore TR, et al. Dopamine agonists and the suppression of impulsive motor actions in parkinson disease. $\mathrm{J}$ Cogn Neurosci. 2012;24:1709-24.

37. Stephens DN, Ripley TL, Borlikova G, Schubert M, Albrecht D, Hogarth L, et al. Repeated ethanol exposure and withdrawal impairs human fear conditioning and depresses long-term potentiation in rat amygdala and hippocampus. Biol Psychiatry. 2005;58:392-400.

38. Worbe Y, Irvine M, Lange I, Kundu P, Howell NA, Harrison NA, Bullmore ET, Robbins TW, Voon V. Neuronal correlates of riskseeking attitudes to anticipated losses in binge drinkers. Biol Psychiatry. 2013.

39. Robinson ES, Eagle DM, Economidou D, Theobald DE, Mar AC, Murphy ER, et al. Behavioural characterisation of high impulsivity on the 5-choice serial reaction time task: Specific deficits in 'waiting' versus 'stopping'. Behav Brain Res. 2009;196:310-6.

40. Cardinal RN, Winstanley CA, Robbins TW, Everitt BJ. Limbic corticostriatal systems and delayed reinforcement. Ann N Y Acad Sci. 2004;1021:33-50.

41. Cardinal RN, Pennicott DR, Sugathapala CL, Robbins TW, Everitt BJ. Impulsive choice induced in rats by lesions of the nucleus accumbens core. Science. 2001;292:2499-501.

42. Winstanley CA, Theobald DE, Cardinal RN, Robbins TW. Contrasting roles of basolateral amygdala and orbitofrontal cortex in impulsive choice. J Neurosci. 2004;24:4718-22. 
43. Ballard K, Knutson B. Dissociable neural representations of future reward magnitude and delay during temporal discounting. Neuroimage. 2009;45:143-50.

44. Tanaka SC, Doya K, Okada G, Ueda K, Okamoto Y, Yamawaki S. Prediction of immediate and future rewards differentially recruits cortico-basal ganglia loops. Nat Neurosci. 2004;7:887-93.

45. Kable JW, Glimcher PW. The neural correlates of subjective value during intertemporal choice. Nat Neurosci. 2007;10: $1625-33$.

46. McClure SM, Laibson DI, Loewenstein G, Cohen JD. Separate neural systems value immediate and delayed monetary rewards. Science. 2004;306:503-7.

47. McClure SM, Ericson KM, Laibson DI, Loewenstein G, Cohen JD. Time discounting for primary rewards. J Neurosci. 2007;27:5796-804.

48. Roesch MR, Taylor AR, Schoenbaum G. Encoding of timediscounted rewards in orbitofrontal cortex is independent of value representation. Neuron. 2006;51:509-20.

49. Mar AC, Walker AL, Theobald DE, Eagle DM, Robbins TW. Dissociable effects of lesions to orbitofrontal cortex subregions on impulsive choice in the rat. J Neurosci. 2011;31:6398-404.

50. Sellitto M, Ciaramelli E, di Pellegrino G. Myopic discounting of future rewards after medial orbitofrontal damage in humans. J Neurosci. 2010;30:16429-36.

51. Reynolds B, Schiffbauer R. Measuring state changes in human delay discounting: an experiential discounting task. Behav Process. 2004;67:343-56.
52. Schweighofer N, Shishida K, Han CE, Okamoto Y, Tanaka SC, Yamawaki S, et al. Humans can adopt optimal discounting strategy under real-time constraints. PLoS Comput Biol. 2006;2:e152.

53. Young JW, Light GA, Marston HM, Sharp R, Geyer MA. The 5choice continuous performance test: evidence for a translational test of vigilance for mice. PLoS One. 2009;4:e4227.

54. Aron AR, Poldrack RA. Cortical and subcortical contributions to stop signal response inhibition: role of the subthalamic nucleus. J Neurosci. 2006;26:2424-33.

55. Aron AR, Fletcher PC, Bullmore ET, Sahakian BJ, Robbins TW. Stop-signal inhibition disrupted by damage to right inferior frontal gyrus in humans. Nat Neurosci. 2003;6:115-6.

56. Swick D, Ashley V, Turken U. Are the neural correlates of stopping and not going identical? Quantitative meta-analysis of two response inhibition tasks. Neuroimage. 2011;56:1655-65.

57. Aron AR. From reactive to proactive and selective control: developing a richer model for stopping inappropriate responses. Biol Psychiatry. 2011;69:e55-68.

58. Verbruggen F, Logan GD. Proactive adjustments of response strategies in the stop-signal paradigm. J Exp Psychol Hum Percept Perform. 2009;35:835-54.

59. Ballanger B, van Eimeren T, Moro E, Lozano AM, Hamani C, Boulinguez P, et al. Stimulation of the subthalamic nucleus and impulsivity: release your horses. Ann Neurol. 2009;66:817-24.

60. Frank MJ, Samanta J, Moustafa AA, Sherman SJ. Hold your horses: impulsivity, deep brain stimulation, and medication in parkinsonism. Science. 2007;318:1309-12. 